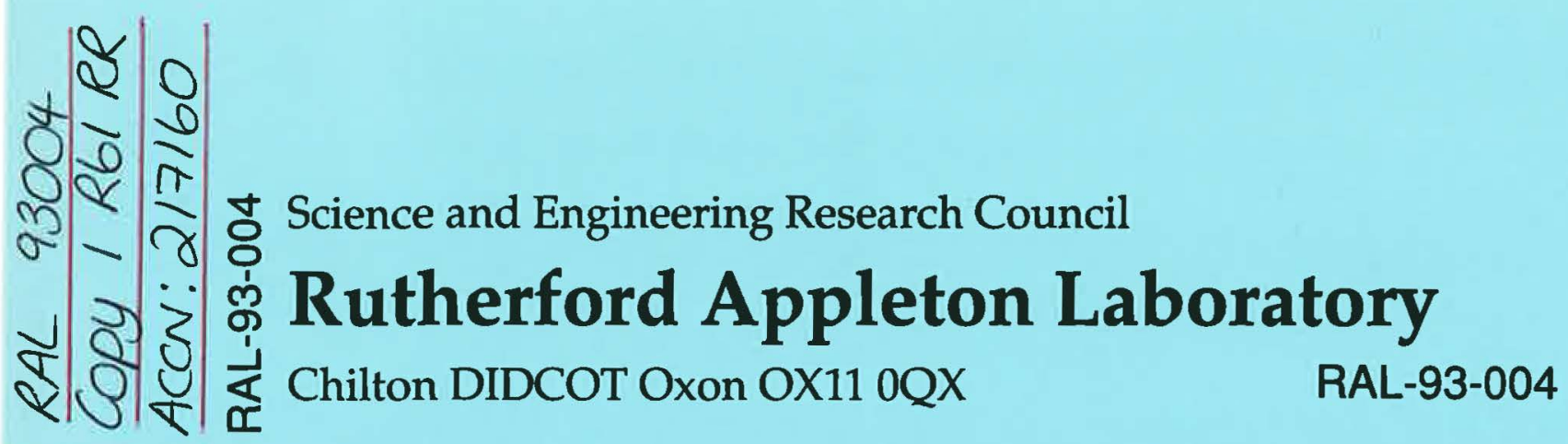

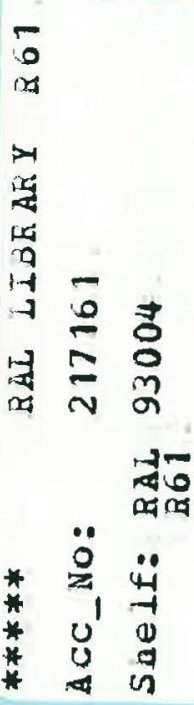

The Spectrum of Longitudinal Spin Fluctuations in a Ferromagnet Including Dipolar and Zeeman Energies

K N Trohidou and S W Lovesey

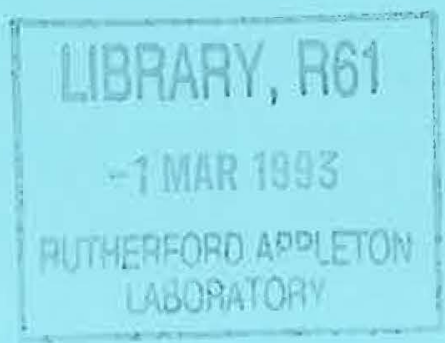

January 1993 
Science and Engineering Research Council

"The Science and Engineering Research Council does not accept any responsibility for loss or damage arising from the use of information contained in any of its reports or in any communication about its tests or investigations" 


\title{
The Spectrum of Longitudinal Spin Fluctuations in a Ferromagnet Including Dipolar and Zeeman Energies
}

\author{
K N Trohidou(1) and S W Lovesey(2)
}

(1) National Centre for Scientific Research, Institute for Materials

Science, "Democritos", P O Box 60228, Athens, Greece.

(2) Rutherford Appleton Laboratory, Oxfordshire, OX11 OQX, UK.

\begin{abstract}
The spectrum of longitudinal spin fluctuations, observed in inelastic neutron scattering experiments, is provided for a Heisenberg model of an ordered. ferromagnet, including dipolar forces and a magnetic field. A numerical method is used to obtain results, from an expression derived with linear spin-wave theory, for wave vectors (k) throughout the Brillouin zone as a function of frequency $(\omega)$, and at various temperatures. Particular attention is given to realistic models of EuO and EuS. Results show that dipolar forces diminish the intensity in the longitudinal response function $S(k, \omega)$ by about a factor of 2 , in accord with previous results for the isothermal susceptibility. The evolution of $S(k, \omega)$ with $\mathbf{k}$ displays several pronounced features, and for EuS there is a strong dependence on the direction of $\mathbf{k}$ for $k$ near the zone boundary.
\end{abstract}

\section{Introduction}

The dearth of experimental data on the spectrum of spin fluctuations along the spontaneous magnetization axis (longitudinal fluctuations) in a ferromagnet is an anomaly when taken in context with the abundant, detailed data available on transverse (spin-wave) excitations in various compounds and metals. By and large, neutron scattering is the preferred experimental method by which to study spin-wave dispersions and lifetimes. The shortage of data on longitudinal fluctuations is caused largely by technical difficulties in extracting this component from the neutron crosssection. A simple scattering geometry exists such as to measure just the transverse spectrum, but the longitudinal spectrum is always measured together with the transverse. In consequence, a differencing method is required to separate the longitudinal and transverse components in data sets, and the quality of data for the longitudinal spectrum is much inferior to that for the directly measured transverse spectrum. Polarization analysis affords in principle a clean separation of the two components, but the apparatus for the method is not yet very efficient. 
Even so, recent progress in studies of the longitudinal spin fluctuation spectrum has been achieved by using polarization analysis. Interestingly, results are not in accord with theory, which dates back to pioneering work on excitations in the Heisenberg ferromagnet; see [1,2] and references therein. If $\chi(\mathbf{k})$ denotes the longitudinal wave vector dependent susceptibility, the prediction is $\chi(\mathbf{k}) \rightarrow(1 / \mathrm{k})$ for $k \rightarrow 0$. The singular behaviour results from the presence of Goldstone bosons in an isotropic Heisenberg magnet. Application of a magnetic field generates a gap in the spectrum (the bosons acquire a mass) and $\chi(0) \sim(1 / \mathrm{H})^{1 / 2}$ where $\mathrm{H}$ is the applied field. These results can be verified from the longitudinal spin response function $\mathbf{S}(\mathbf{k}, \omega)$ using the standard relation

$$
\chi(\mathbf{k})=\int_{-\infty}^{\infty} \mathrm{d} \omega\{\mathrm{S}(\mathbf{k}, \omega) / \omega\}
$$

and the result, obtained for non-interacting spin waves with dispersion $\mathrm{E}_{\mathrm{K}}=\mathrm{Dk}^{2}$,

$$
\begin{aligned}
& S_{o}(k, \omega)=\left(\frac{T_{0} k^{3}}{16 \pi^{2} E^{2} k}\right)\{1+n(\omega)\}^{*} \\
& \ln \left\{1+\left(\{1+n(\omega)\}\left\{\exp \left[\left(4 h E_{k}+\left(\omega-E_{k}\right)^{2}\right) / 4 E_{k} T\right]-1\right\}\right)^{-1}\right\}
\end{aligned}
$$

where $v_{0}$ is the volume of a unit cell, $h=g \mu_{B} H$ is the Zeeman energy, and (Boltzmann's constant $=1$ )

$$
\mathrm{n}(\omega)=\{\exp (\omega / \mathrm{T})-1\}^{-1}
$$

In the limit $k \rightarrow 0$ ( $\omega$ fixed) $S_{0}(k, \omega)$ vanishes, while for $\omega \rightarrow 0$ (k fixed) it achieves a value which increases with decreasing $k$. The peak in $S_{0}(k, \omega)$ at the spin wave energy $\omega=E_{k}$ is suppressed by the Zeeman energy, and it coalesces with the quasielastic response when $k$ is decreased and $E_{k} \rightarrow 0$. The frequency dependence of $S_{0}(k, \omega)$ is illustrated in fig (1). Observe that $(1.2)$ is the density fluctuation response for a Bose fluid with a chemical potential $=-h$.

The discrepancy between measured results and theoretical predictions, for response functions of an isotropic Heisenberg magnet, has prompted investigations of the influence on static and dynamic response functions of the usually very weak dipolar interactions. It has been shown that, dipolar interactions, present to some extent in all magnets, reduce the magnitude of $\chi(\mathbf{k})$ but leave unchanged the divergence at long wavelengths [1]. The contribution to the longitudinal response function induced by dipolar forces is significantly different from $S_{0}(k, \omega)$, at least at 
long wavelengths where it stems from two spin-wave creation events, which contribute even at absolute zero temperature [2]. The dipolar induced response vanishes below,

$$
\omega_{0}=2 \sqrt{ }\left(h^{2}+2 \varepsilon h\right)
$$

where $\varepsilon$ determines the strength of the dipolar forces (see Table 1), and it decreases rapidly with increasing $\omega$.

In this paper we provide, for the first time, a comprehensive survey of the longitudinal dynamic response function $S(k, \omega)$ including the influences of dipolar forces, Zeeman energy and temperature. Results are given for wave vectors out to the zone boundary. The definition of $S(k, \omega)$ is reviewed in the next section. While the result used in our calculations is based on linear (non-interacting) spin-wave theory it might be useful quite close to the critical temperature, $T_{c}$, since the lowest order processes that contribute to $S(\mathbf{k}, \omega)$ involve two spin-wave events.

The numerical method for calculating $S(\mathbf{k}, \omega)$, for arbitrary $\mathbf{k}$ and $\omega$, is described in $\S 3$. Results for realistic models of EuO and EuS are gathered in $\S 4$, and $\$ 5$ contains a discussion of our findings.

\section{Spin-wave Expression for $S(k, \omega)$}

The longitudinal spin response function $S(\mathbf{k}, \omega)$ is the spatial and temporal Fourier transforms of the correlation function $\left\langle S_{I}^{Z} S_{m}^{Z}(t)\right\rangle$, where $S_{m}^{Z}$ is the spin component at the lattice site defined by $m$ parallel with the spontaneous magnetization ( $\mathrm{z}$ - axis). In linear spin-wave theory, it is adequate to use for $S^{z}$ the approximate relation [3],

$$
S^{2}=S-S^{-} S^{+} / 2 S
$$

where $S$ is the magnitude of the spin, and $S^{ \pm}$are standard spin raising and lowering operators. It follows that $S(\mathbf{k}, \omega)$ is the sum of two components, one that vanishes unless $\omega=0$, and a second nontrivial component derived from,

$$
(1 / 2 S)^{2}\left\langle S^{-} S^{+} S^{-}(t) S^{+}(t)\right\rangle
$$

In linear spin-wave theory, $\mathrm{S}^{-} \sim \mathrm{a}^{+}$where $\mathrm{a}, \mathrm{a}^{+}$are Bose operators; hence $(2.2)$ is of the form of a particle density auto-correlation function, c.f. (1.2). 
The full calculation of $S(\mathbf{k}, \omega)$ using linear spin-wave theory for a Heisenberg ferromagnet including dipolar and Zeeman energies is provided by Lovesey and Trohidou [1]. Here we limit ourselves to just recording the final expression, namely,

$$
\begin{aligned}
& \mathrm{S}(\mathbf{k}, \omega)=\{1+\mathrm{n}(\omega)\} \frac{1}{\mathrm{~N}} \sum_{p, q} \delta_{\mathbf{p}-\mathbf{q}, \mathbf{k}}\left\{\frac{1}{2} \mathrm{E}(\mathbf{p}, \mathbf{q})\left(1+\mathrm{n}_{\mathbf{p}}+\mathrm{n}_{\mathbf{q}}\right)\right. \\
& \left.*\left[\delta\left(\omega-\mathrm{E}_{\mathbf{p}}-\mathrm{E}_{\mathbf{q}}\right)-\delta\left(\omega+\mathrm{E}_{\mathbf{p}}+\mathrm{E}_{\mathbf{q}}\right)\right]+\mathrm{F}(\mathbf{p}, \mathbf{q})\left(\mathrm{n}_{\mathbf{p}}-\mathrm{n}_{\mathbf{q}}\right) \delta\left(\omega+\mathrm{E}_{\mathbf{p}}-\mathrm{E}_{\mathbf{q}}\right)\right\}
\end{aligned}
$$

In this expression, the wave vectors $\mathbf{p}, \mathbf{q}$ are restricted to a Brillouin zone, and $n_{\mathbf{p}}$ is the Bose distribution function for a spin-wave with energy $E_{p}$ (the result $E_{p}=D p^{2}$ applies in the limit of long wavelengths for a simple isotropic ferromagnet). The structure factors $E(\mathbf{p}, \mathbf{q})$ and $F(\mathbf{p}, \mathbf{q})$ can be shown to satisfy $E, F>, 0$, and $E$ vanishes while $F \rightarrow 1$ in the absence of dipolar forces; explicit expressions are given in [1]. In the absence of dipolar forces, $S(\mathbf{k}, \omega)$ is generated by event that involve creation and annihilation of spin-waves, as expected for density fluctuations, i.e. $\mathbf{a}_{\mathbf{q}}^{+}(\mathrm{t}) \mathbf{a}_{\mathbf{p}}^{-}(\mathrm{t})$. Dipolar forces induce off-diagonal fluctuations, of the form $\mathrm{a}_{\mathbf{q}}(t) \mathrm{a}_{\mathbf{p}}(\mathrm{t})$, as well as contributing to the spin-wave energy and $F(p, q)$. The result (1.2) is obtained from (2.3) on taking $E=0, F=1$, and using $E_{k}=h+D k^{2}$ which is appropriate for long wavelength spin fluctuations.

When $\mathrm{k} \sim(\varepsilon / \mathrm{D})^{1 / 2} \sim 0$ the contribution to $\mathrm{S}(\mathrm{k}, \omega)$ induced by dipolar forces is, to a good approximation $(\omega \geq 0)$,

$$
S_{d}(k \sim 0, \omega)=\{1+n(\omega)\}(1 / 2 N) \sum_{\mathbf{p}} E(p, p)\left(1+2 n_{p}\right) \delta\left(\omega-2 E_{p}\right)
$$

It can be shown that $S_{d}(k \sim 0, \omega)$ vanishes for $0<\omega<\omega_{0}$, where $\omega_{0}$ is given by (1.4) [2]. In the limit of small magnetic fields, $h \rightarrow 0$,

$$
\mathrm{S}_{\mathrm{d}}\left(\mathrm{k} \sim 0, \omega_{\mathrm{o}}\right) \sim\left(\mathrm{T}^{2} / \mathrm{he}^{1 / 2}\right), \mathrm{h} \ll \mathrm{e} .
$$

Hence, the dipolar contribution to the longitudinal response is large for small magnetic fields. 


\section{Numerical Method}

The longitudinal response function $S(k, \omega)$ has been evaluated numerically for a range of parameters. The summation in eq (2.3) was done over a mesh of evenly spaced points in the Brillouin zone. For values of $\mathrm{k}$ along symmetry directions the numerical procedure could be made more efficient by restricting the summation to a reduced region of $\mathrm{k}$-space.

For a general $\mathrm{k}$ value however, $6.4 \times 10^{7}$ points in $\mathrm{k}$-space were sufficient to eliminate spurious structure in $S(k, \omega)$ due to the finite mesh size. Frequency was sampled in intervals of $\omega \simeq 0.03 \mathrm{meV}$. The magnetic field is parallel to the easy axis, which is $(1,1,1)$ for both EuO and EuS. The wave vector $\mathbf{k}$ is specified with respect to this axis and $(\xi, 0,0),(0, \xi, 0)$ and $(0,0, \xi)$ are respectively parallel to $(-1,-1,2),(1,-1,0)$ and $(1,1,1)$ in real space cubic axis.

To assess the accuracy of our numerical procedure we compare our numerical results for a very small value of $\mathrm{k}$ with the analytically exact ones given by eq (1.2). This comparison is given in fig. (2). The solid line is giving $S(k, \omega)$ for EuO without the dipolar forces for $\mathrm{k}=0.1(\pi / \mathrm{a}) \AA^{-1}$, temperature $\mathrm{T}=0.2 \mathrm{~T}_{\mathrm{C}}$ in the presence of a magnetic field $H=0.1 T$ and the dashed line the results for $S(k, \omega)$ for the same value of $\mathrm{k}, \mathrm{T}$ and $\mathrm{H}$ using equation (1.2). The good agreement between the two sets of results shown in fig. (2) gives us confidence in our numerical method.

\section{Results}

Values of $\mathbf{S}(\mathbf{k}, \omega)$ defined in eq (2.3) and reference [1] have been obtained, for realistic models of EuO and EuS, using the numerical method described in $\$ 3$. Parameters for these two ferromagnets are gathered in Table 1. The dipolar energy parameter $\varepsilon$ that appears in eq (1.4) is defined to be

$$
\varepsilon=2 \pi \mathrm{g} \mu_{\mathrm{B}} \mathrm{M}_{\mathrm{o}}
$$

where $\mathrm{M}_{0}$ is the saturation magnetization. The spin wave energy $\omega_{\mathbf{k}}$ satisfies,

$$
\omega_{k}^{2}=\left(E_{k}+h\right)\left(E_{k}+h+2 \varepsilon \sin ^{2} \theta_{k}\right)
$$

in which $\theta_{\mathbf{k}}$ is the angle between $\mathbf{k}$ and the easy axis, and $E_{\mathbf{k}}$ is the exchange energy derived from nearest $\left(J_{1}\right)$ and next nearest $\left(J_{2}\right)$ shells of magnetic ions, that has the 
form $\mathrm{Dk}^{2}$ in the limit of long wavelengths $(\mathrm{ak}<<1)$. Note that, for a fcc lattice the spin wave stiffness

$$
\mathrm{D}=a^{2} 2 \mathrm{~S}\left(\mathrm{~J}_{1}+\mathrm{J}_{2}\right)
$$

and for europium ions $S=7 / 2$.

Regarding the Zeeman energy $\mathrm{h}=\mathrm{g} \mu_{\mathrm{B}} \mathrm{H}$ it is useful to note that for a field $\mathrm{H}=1 \mathrm{~T}$ and $\mathrm{g}=2.0$, we have $\mathrm{h}=0.116 \mathrm{meV}$. At high temperature $\left(\mathrm{T} \geq 0.8 \mathrm{~T}_{\mathrm{C}}\right)$ the dipolar contribution to the spin dynamics can be safely mimicked by an effective magnetic field. It has been shown [5] that in this temperature range the dipolar energy in the spin wave energy is adequately represented by use of the expression

$$
\omega_{k}=\left(E_{k}+h+\frac{2}{3} \varepsilon\right)
$$

The last term in this expression, obtained by averaging the dipolar contribution in (4.2) over the directions of $\mathbf{k}$, is just an addition to the Zeeman energy, i.e. an effective Zeeman energy equal to $(h+2 \varepsilon / 3)$.

To set the scene for our findings for $S(k, \omega)$ over a wide range of $\mathbf{k}$, and including the dipolar energy, we refer to fig. (1). Here, we illustrate the frequency dependence of the longitudinal response at long wavelengths of a simple ferromagnet (no dipolar forces). The results are derived from eq (1.2). The intensity of the spin wave excitation at $\omega \sim \mathrm{E}_{\mathrm{k}}$ is determined by the Zeeman energy, and there is no significant structure in the response near $\omega \sim-E_{k}$. In all other figures we report data only for $\dot{\omega} \geq 0$. Note that the function in (1.2) has a first derivative with respect to $\omega$ which is positive -definite at $\omega=0$ for all values of the parameters.

Calculations reported in the balance of this section have been made for a field $\mathrm{H}=0.1 \mathrm{~T}$, so $\mathrm{h}=0.01 \mathrm{meV}$. It is useful to bear in mind values of $(2 \varepsilon / 3)$ for EuO and EuS, namely, 0.09 and $0.06 \mathrm{meV}$, respectively. For both materials, the dipolar energy is therefore large compared to the Zeeman energy. All the calculations that include dipolar forces are made with eq (2.3) and not the simplified treatment, appropriate at only high temperatures, in which they are represented by an effective magnetic field of magnitude (2ع/3).

The results of calculations of the response function $S(k, \omega)$ in the presence of a magnetic field $\mathrm{H}=0.1 \mathrm{~T}$ are shown in figs 3-4 for several values of $\mathbf{k}$ in the $(1,0,0)$ direction less than or equal to the zone boundary for temperatures $\mathrm{T}=0.4$ and $0.8 \mathrm{~T}_{\mathrm{c}}$. $S(k, \omega)$ is in units of $\mathrm{eV}^{-1}$, the solid lines give the response function without the 
dipolar forces and the dashed lines give $S(k, \omega)$ including dipolar forces. The magnitude of the wave vector $\mathrm{k}$ is in units ( $\pi / \mathrm{a})$ which is $0.61,0.53 \AA^{-1}$ for EuO and EuS, respectively.

\section{EuO}

As we can see from fig 3, for a small value of the wave vector there is a spin-wave peak in $S(k, \omega)$ around the value predicted from the spin wave theory $(\omega=$ $\mathrm{Dk}^{2}+\mathrm{h}=1.10 \mathrm{meV}$ for $\mathrm{k}=0.5$ in fig $3 \mathrm{a}$ ). The diminution of intensity at $\omega=0$ fades with increasing k, c.f. fig. 1 and $3 b-3 d$. The influence of the dipolar forces is to decrease the response function, and suppress features in the intensity distribution including the spin-wave peak. This finding is consistent with the notion that, in some respects, dipolar forces have an effect which is akin to an external magnetic field; some of the important differences to be borne in mind are that an external field creates a gap in the spin wave spectrum, and it does not break the conservation of the total magnetization (in the direction of the field).

Moving to larger values of $k$, the results displayed in figs $3 b-3 d$ reveal the appearance of a structured quasi-elastic peak, in contrast to a diminution of intensity for smaller wave vectors, and a pronounced peak at finite $\omega$. The latter is not associated with excitation of a single spin-wave, at least near the zone boundary where it arises simply from a cut-off in intensity imposed by the delta functions in (2.3). It is this feature of the spectrum that is most severely affected by dipolar forces, and relatively more so than the spin-wave peak in the spectrum for the smallest wave vector.

Figs $4 \mathrm{a}$ and $4 \mathrm{~b}$ show the influence of temperature on $S(\mathbf{k}, \omega)$ with and without dipolar forces. As a rough guide, the intensity being derived from two spin wave events might be expected to vary as the square of the temperature, i.e. a factor 4 difference in the two plots in each figure. With regard to the high frequency peak in the spectra, this is an over estimate and the factor is more like 2.7.

An investigation of the dependence of $S(k, \omega)$ on the direction of $\mathbf{k}$ revealed an anticipated behaviour. For small $\mathrm{k}$ the spectrum is almost isotropic, whereas for $\mathbf{k}$ at the zone boundary the slight variation stems from changes in the cut-offs imposed by the delta functions in (2.3). In this respect, EuS, to which we next turn, is more interesting. 
Results shown in fig. 5 are to be compared with the corresponding data for EuO displayed in fig. 3. The main differences between the two sets of results arise from a change in effective energy scales, brought about by the competing nature of the exchange interaction in EuS, e.g. $\mathrm{T}_{\mathrm{c}}$ for EuO and EuS is in the ratio 4.2:1, c.f. Table 1. Thus, we find the width of $\mathbf{S}(\mathbf{k}, \omega)$ for EuS is much less than for EuO; in consequence, structure is less pronounced in the relatively compressed spectrum, but the maximum intensities are larger.

Another effect of the competing interactions in EuS is pronounced spatial anisotropy. This feature is illustrated in fig. 6 which shows $S(k, \omega)$ with $k=(2 \pi / a)$ and $\mathbf{k}$ directed along the three principal directions (relative to the easy-axis). Once more, we find the main effect of dipolar forces is to diminish the intensity of $\mathbf{S}(\mathbf{k}, \omega)$, rather than modify the distributions of intensity.

\section{Discussion}

Our comprehensive investigation of the influence of dipolar forces on the longitudinal dynamical response function $S(\mathbf{k}, \omega)$ shows that the main effect, for both EuO and EuS, is a reduction in intensity by roughly a factor of two. It seems that no new structure in $\mathbf{S}(\mathbf{k}, \omega)$ results from the dipolar forces. These findings are in accord with previous results for the isothermal susceptibility, which is formally the inverse frequency moment of $S(\mathbf{k}, \omega)$, eq (1.1).

There is a rich structure in $\mathbf{S}(\mathbf{k}, \omega)$ with variation of $\mathbf{k}$. For small $\mathbf{k}$, the quasi-elastic peak is weak and of an intensity approximately equal to the spin-wave contribution (in a finite field). The result for a simple magnet, illustrated in fig (1) and appropriate for a small $\mathrm{k}$, never displays more than one peak. In the opposite extreme, when $\mathbf{k}$ is at the zone boundary, the quasi-elastic response is more intense than the high frequency contribution. For a large $\mathrm{k}$, the latter is not attributable to the excitation of a spin-wave, but arises from a cut-off in intensity created by the energy conserving delta functions in the spin-wave formula for $S(\mathbf{k}, \omega)$.

Let us consider the effect of non-linear spin-wave events. These represent spin-wave fluctuations that become more important as $\mathrm{T} \rightarrow \mathrm{T}_{\mathrm{c}}$. In consequence, including non-linear events increases $\chi(\mathbf{k})$ beyond the values obtained from simple (linear) spin-wave theory, and the increase becomes more pronounced as the critical temperature is approached [6]. Near $T_{c}$ the dipolar forces can be mimicked by an effective magnetic field which will, of course, suppress spin fluctuations. In conclusion, for given parameters, results provided here are consistent with a lower bound on $\chi(\mathbf{k})$ (the integrated intensity). 
Table 1. Parameters for EuO and EuSa (FCC, easy axis $(1,1,1)$ )

$\begin{array}{ccccc}\mathrm{J}_{1} & \mathrm{~J}_{2} & \mathrm{D} & \mathrm{M}_{\mathrm{o}} & \varepsilon \\ (\mathrm{K}) & (\mathrm{K}) \quad\left(\mathrm{meV} \AA^{2}\right) & (\mathrm{G}) & (\mathrm{meV}) \\ \mathrm{EuO}(\mathrm{a}=5.14 \AA & \left.\AA, \mathrm{T}_{\mathrm{c}}=69.5 \mathrm{~K}\right) \\ 0.61 & 0.12 & 11.65 & 1910 & 0.14 \\ \operatorname{EuS}\left(\mathrm{a}=5.95 \AA, \mathrm{T}_{\mathrm{C}}=16.5 \mathrm{~K}\right) & & \\ 0.24 & -0.12 & 2.56 & 1184 & 0.09\end{array}$

a After refs. [3,4] 


\section{Figure Captions}

1. The frequency dependence of $S_{O}(k, \omega)$ defined in (1.2) is illustrated for $h=0.01$, $\mathrm{T}=0.5$ and two values of $\mathrm{E}_{\mathrm{k}}=0.2$ and 1.0; these parameters are in units of $\mathrm{meV}$.

2. Results from $S(k, \omega)$ obtained from the analytic expression eq (1.2), derived for a spin wave dispersion $\mathrm{Dk}^{2}$, and a numerical evaluation of the full form given in eq (2.3) are shown for $\mathrm{EuO}$ and a small wave vector, $\mathrm{k}=0.1(\pi / \mathrm{a})$. The other parameters are $\mathrm{H}=0.1 \mathrm{~T}$ and $\mathrm{T}=0.25 \mathrm{~T}_{\mathrm{c}}$.

3. $S(k, \omega)$ for EuO with $\varepsilon=0$ and $0.14 \mathrm{meV}$ is displayed for four values of $\mathbf{k}$ as a function of $\omega ; \mathrm{H}=0.1 \mathrm{~T}$ and $\mathrm{T}=0.4 \mathrm{~T}_{\mathrm{c}}$.

4. The influence of temperature on $S(k, \omega)$ for EuO is shown for $\mathbf{k}=(1.5,0,0)$ with and without dipolar forces.

5. Comparison data to fig. 3 is shown for EuS; $\varepsilon=0$ and $0.09 \mathrm{meV}$.

6. $S(k, \omega)$ for EuS with $\varepsilon=0$ and $0.09 \mathrm{meV}$ is displayed for three different directions of $k$ with $k=2 \pi / a ; T=0.4 T_{c}$ and $H=0.1 T$.

\section{References}

[1] Lovesey, S. W. and Trohidou, K. N. (1991) J. Phys.: Condens. Matter 3, 1827 erratum ibid 3, 5255

[2] Lovesey, S.W. (1991) Europhys. Lett., 15, 63

[3] Keffer, F. (1966) Handbook of Physics vol. XV111/2 (Berlin: Springer)

[4] Passell, L., Dietrich, O.W. and Als-Nielsen, J. (1976) Phys. Rev. B14, 4897

[5] Lovesey, S.W., Karlsson, E.B. and Trohidou, K. N. (1992)

J. Phys.: Condens. Matter 4, 2043

[6] Gaia Pedrolli, University of Florence, private communication

Acknowledgements to the EEC 
$N$

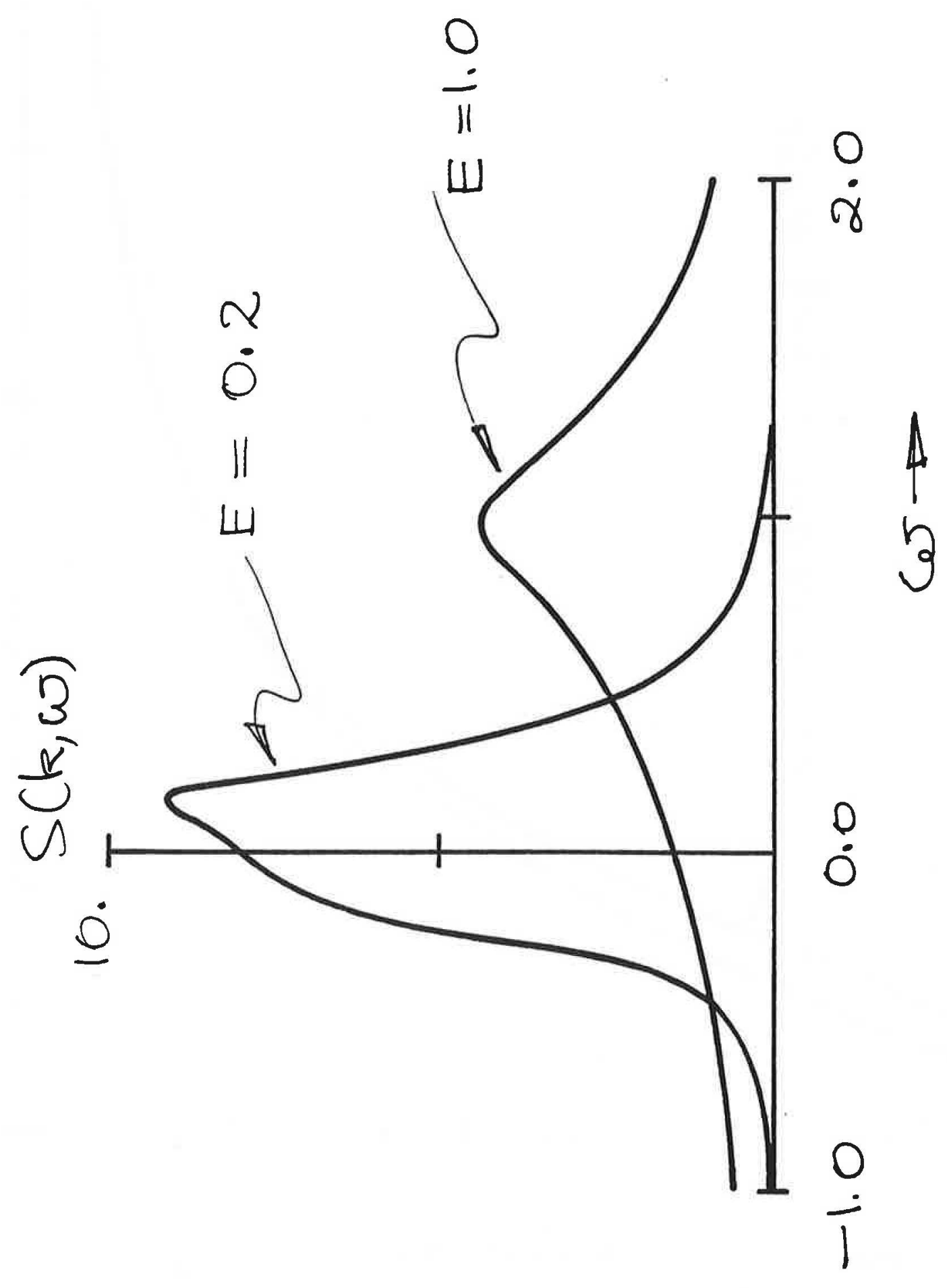


1

(8)

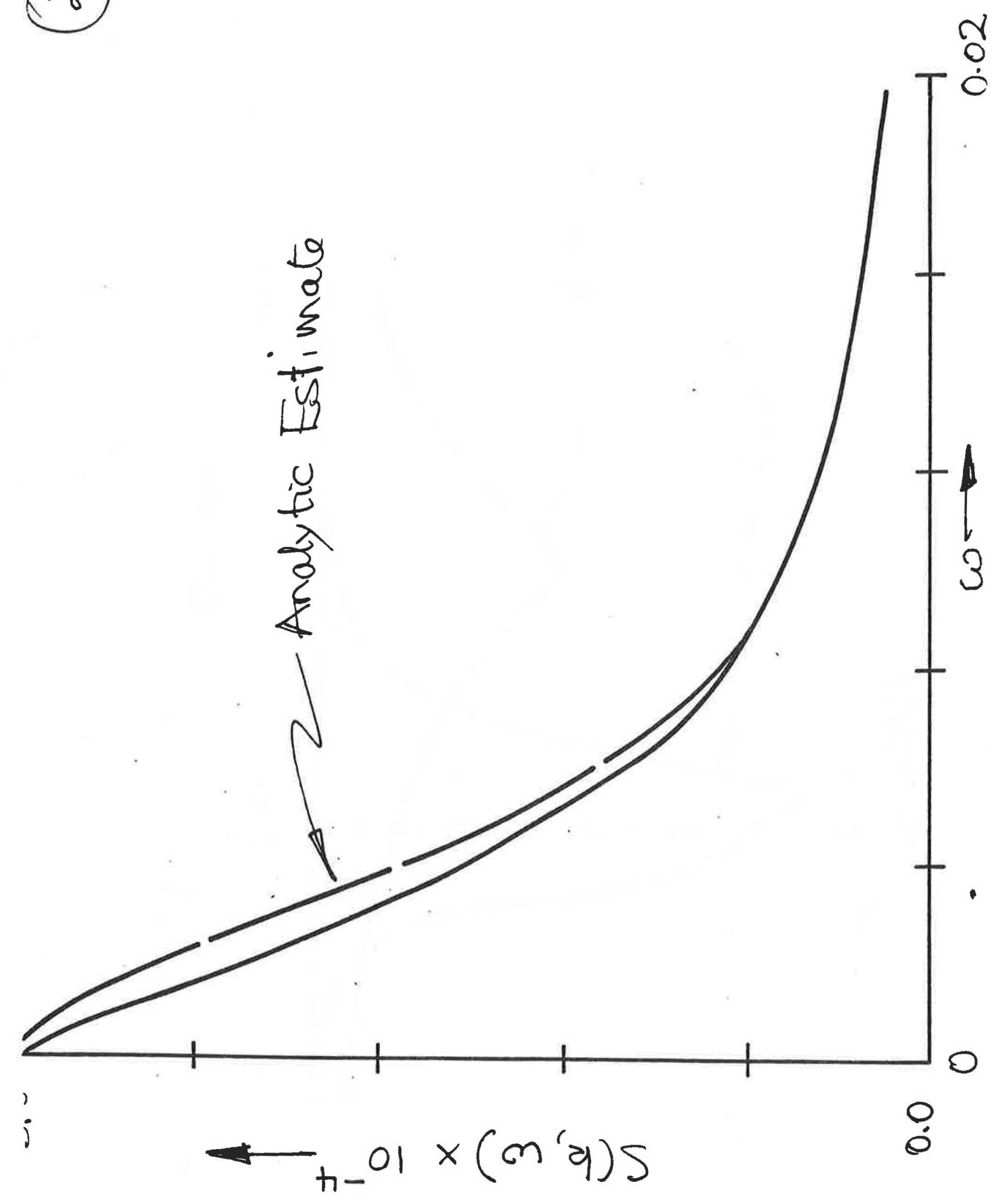


$(\infty)$

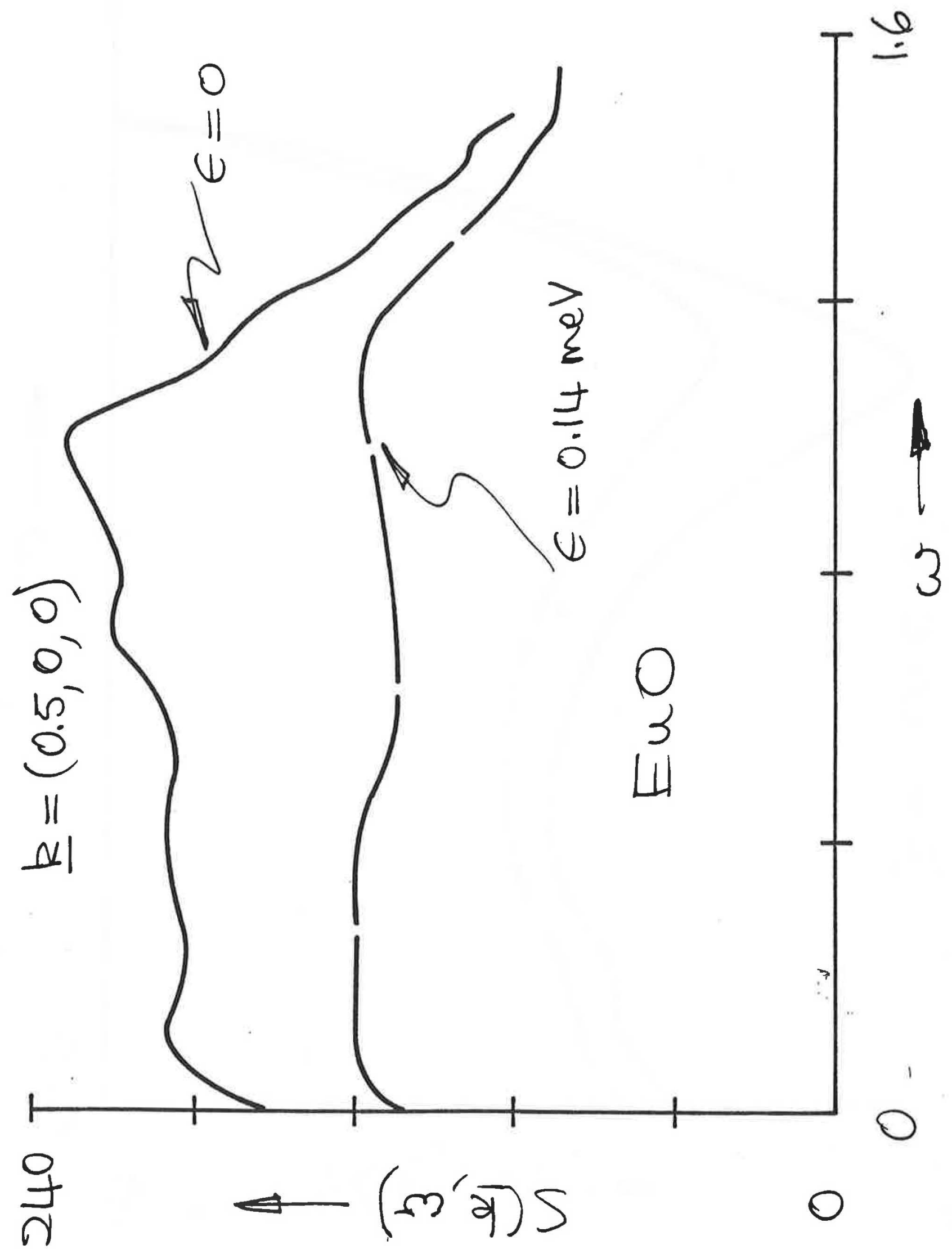




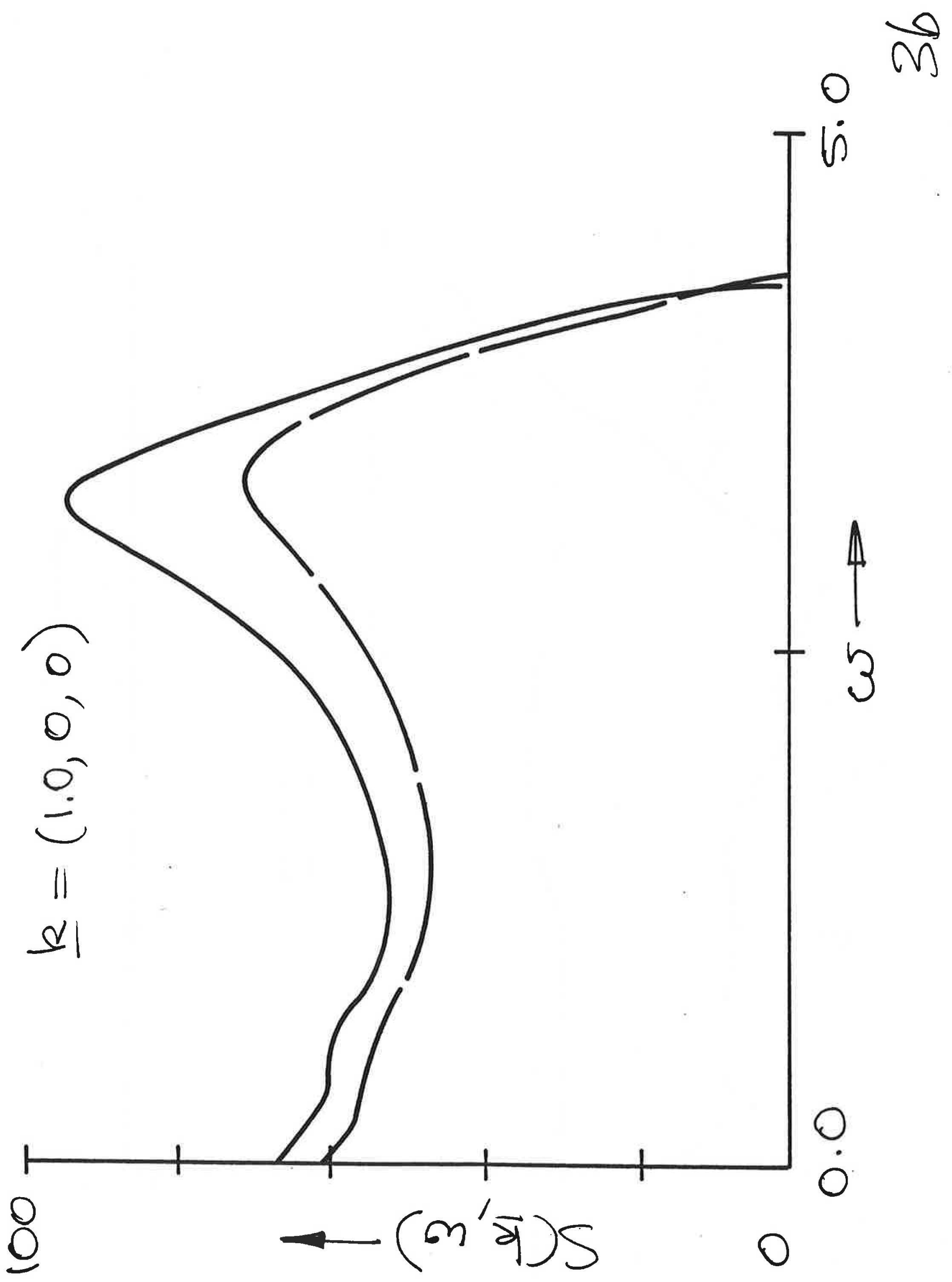


m

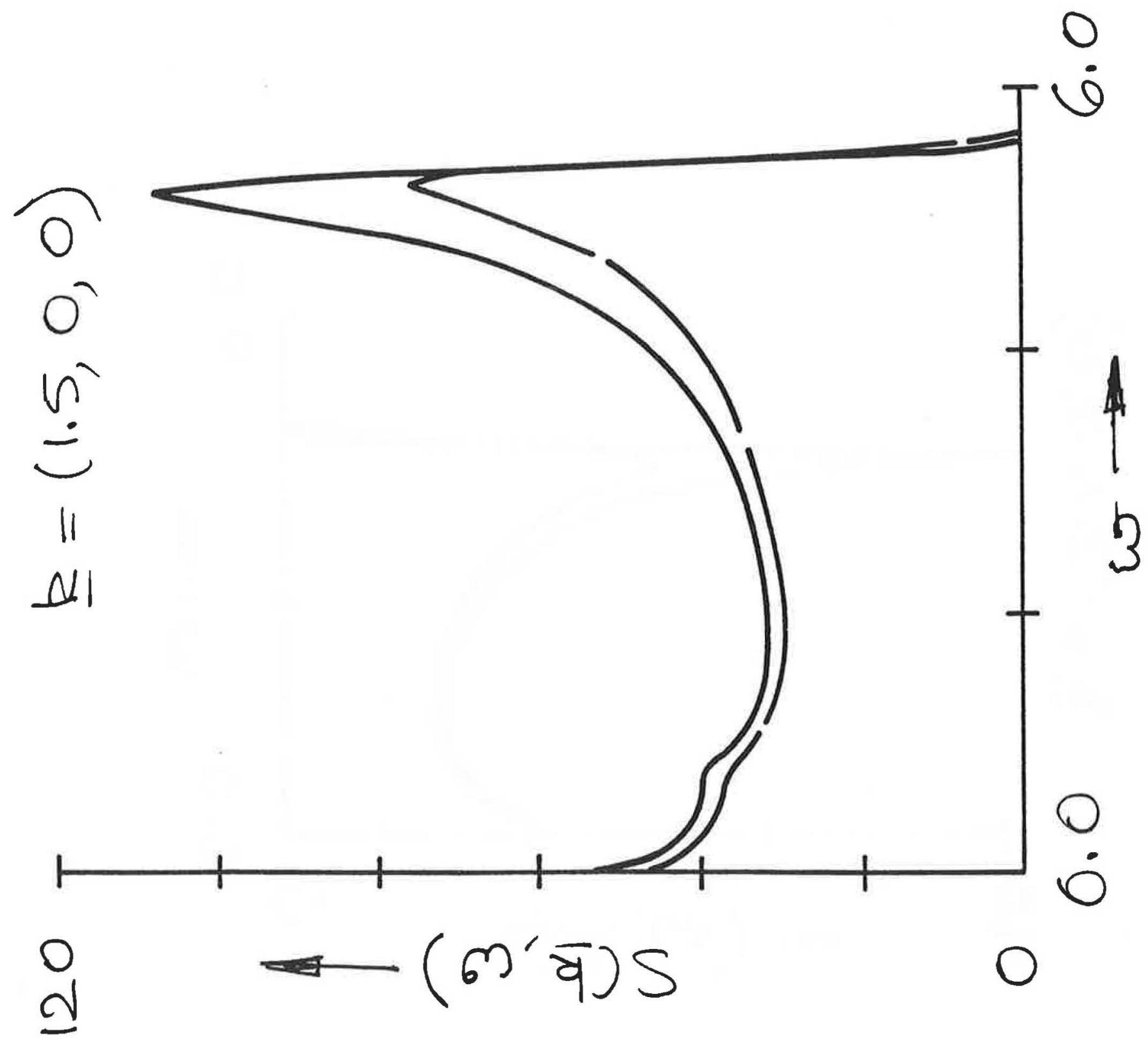




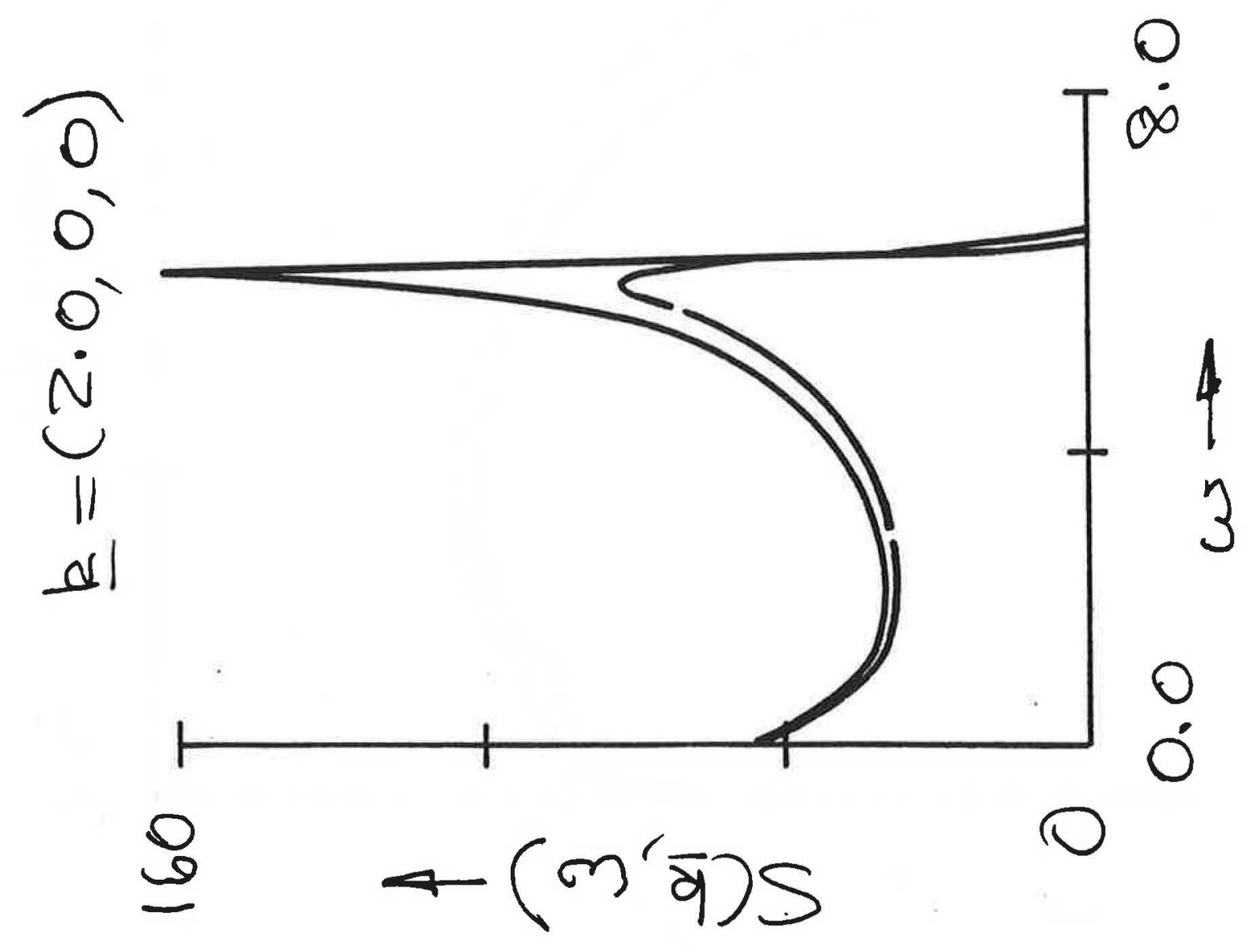




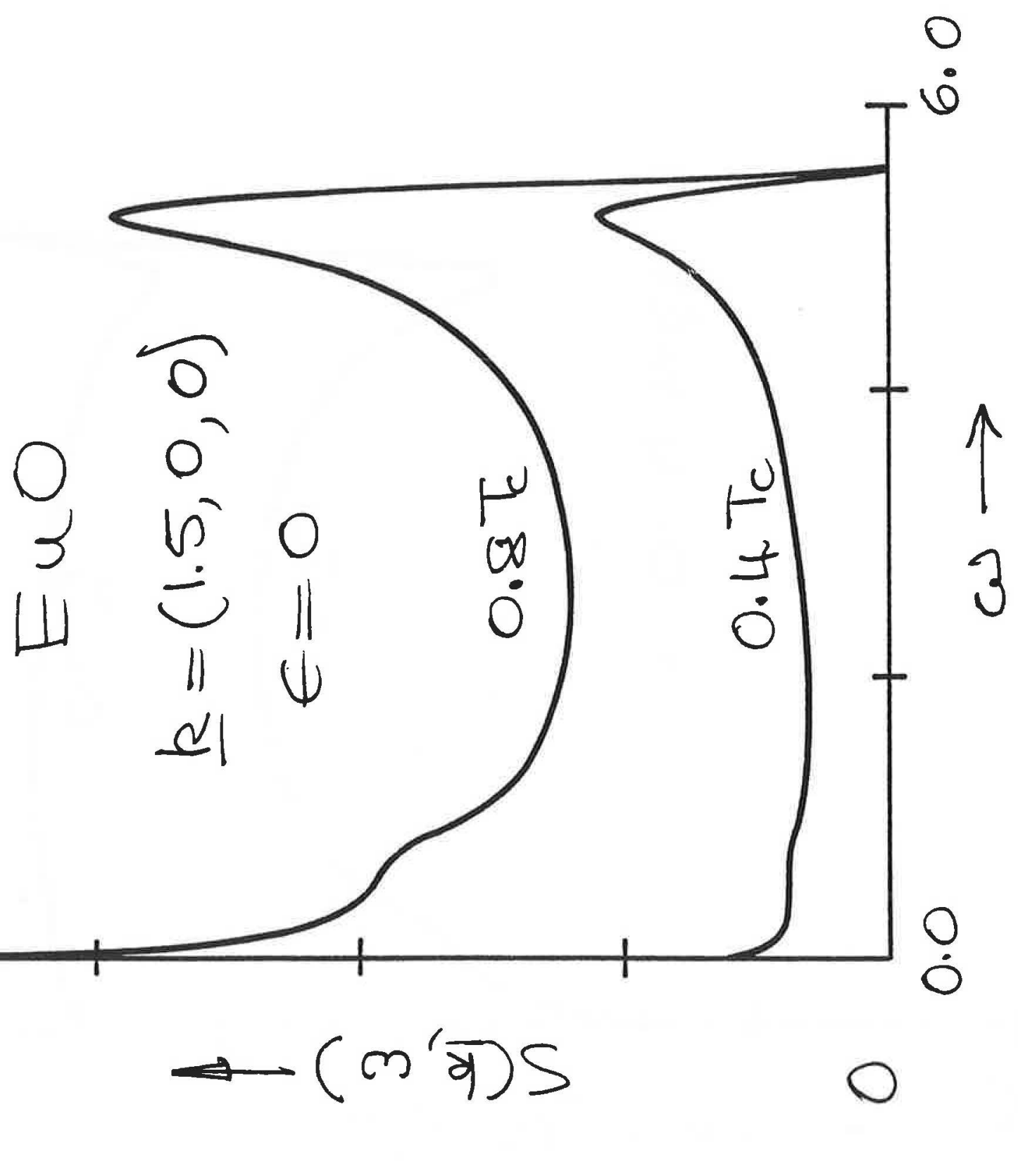


(7)

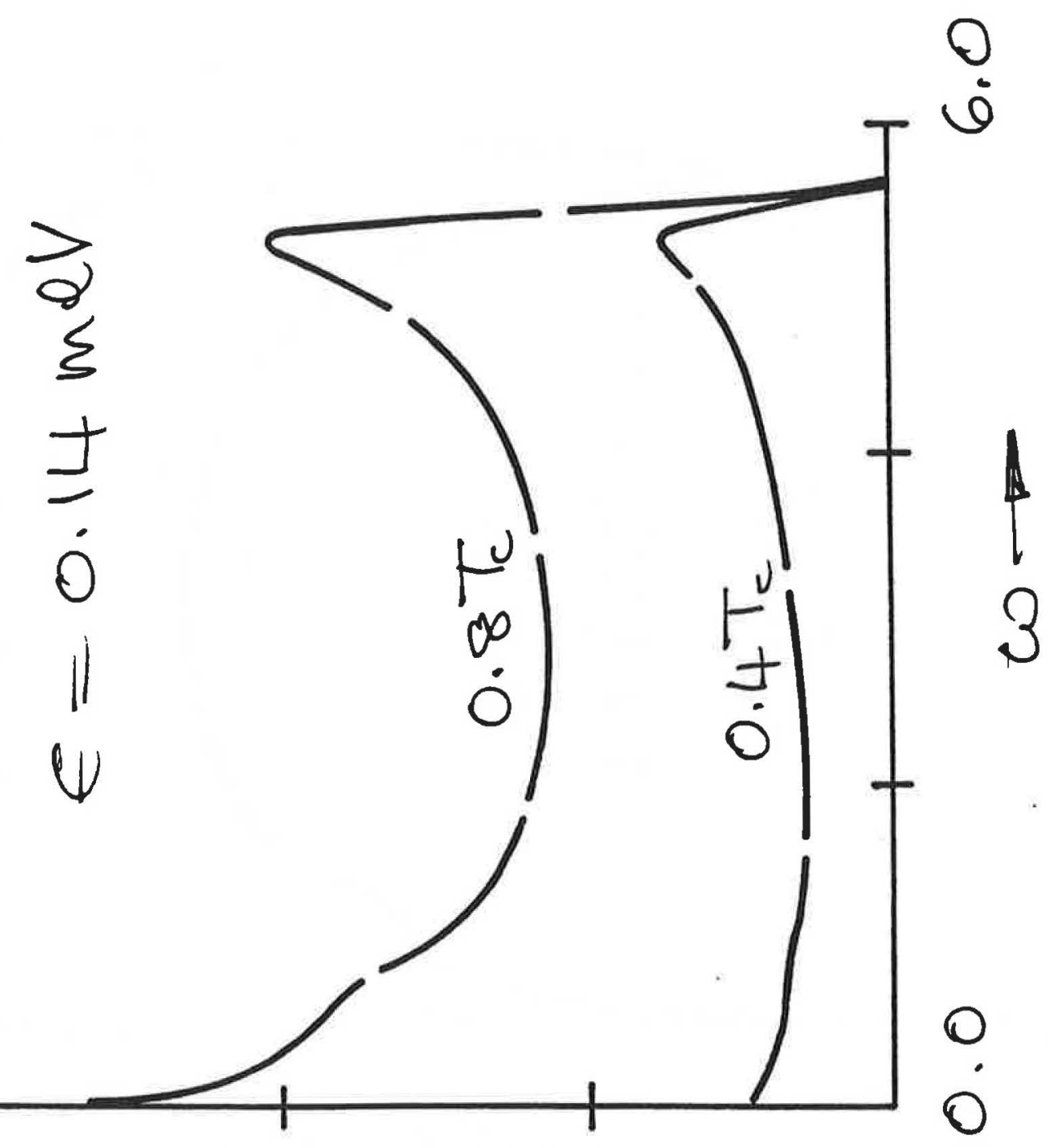

8

$\rightarrow\left(m^{\prime} \bar{y}\right) S$

P 


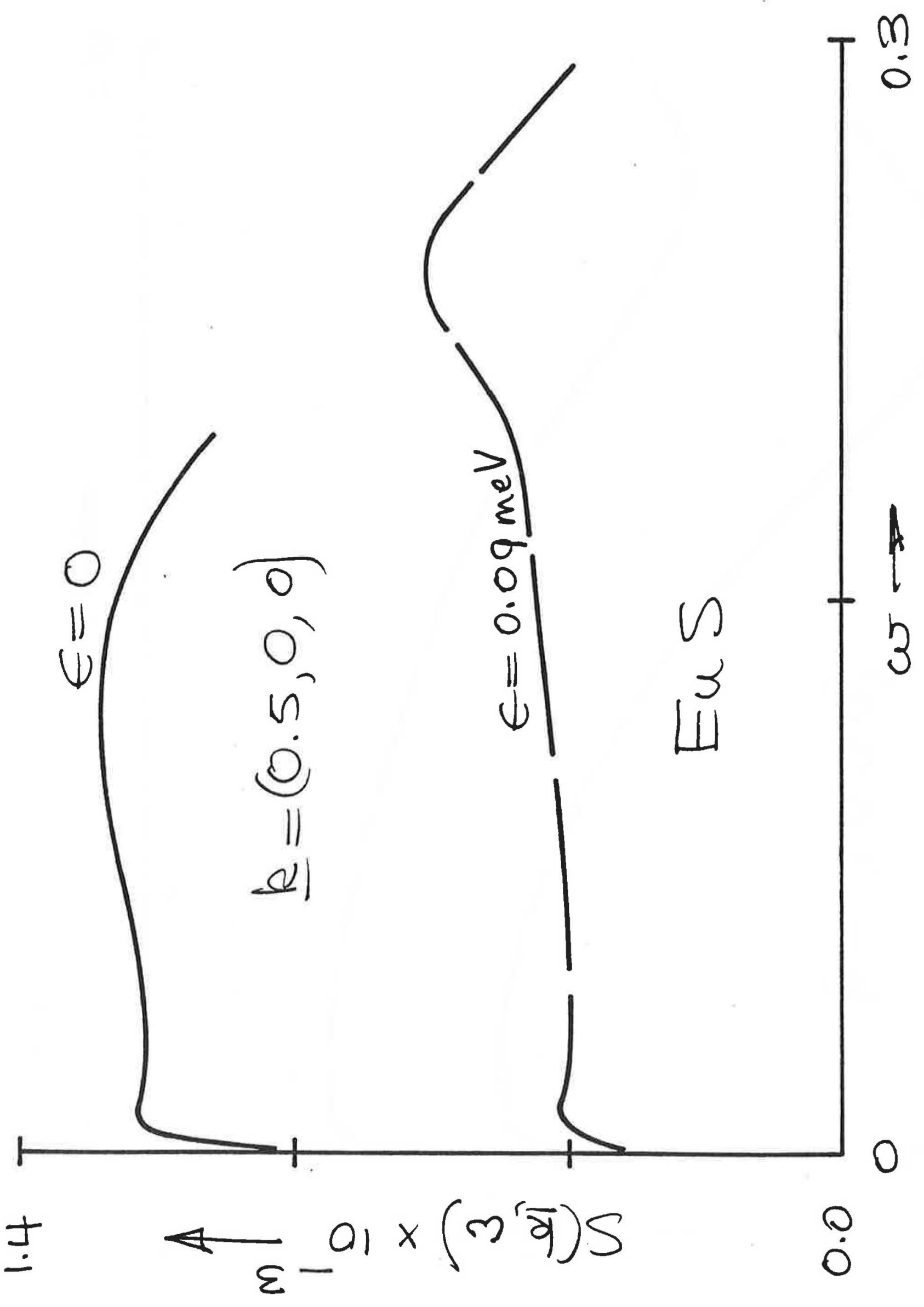




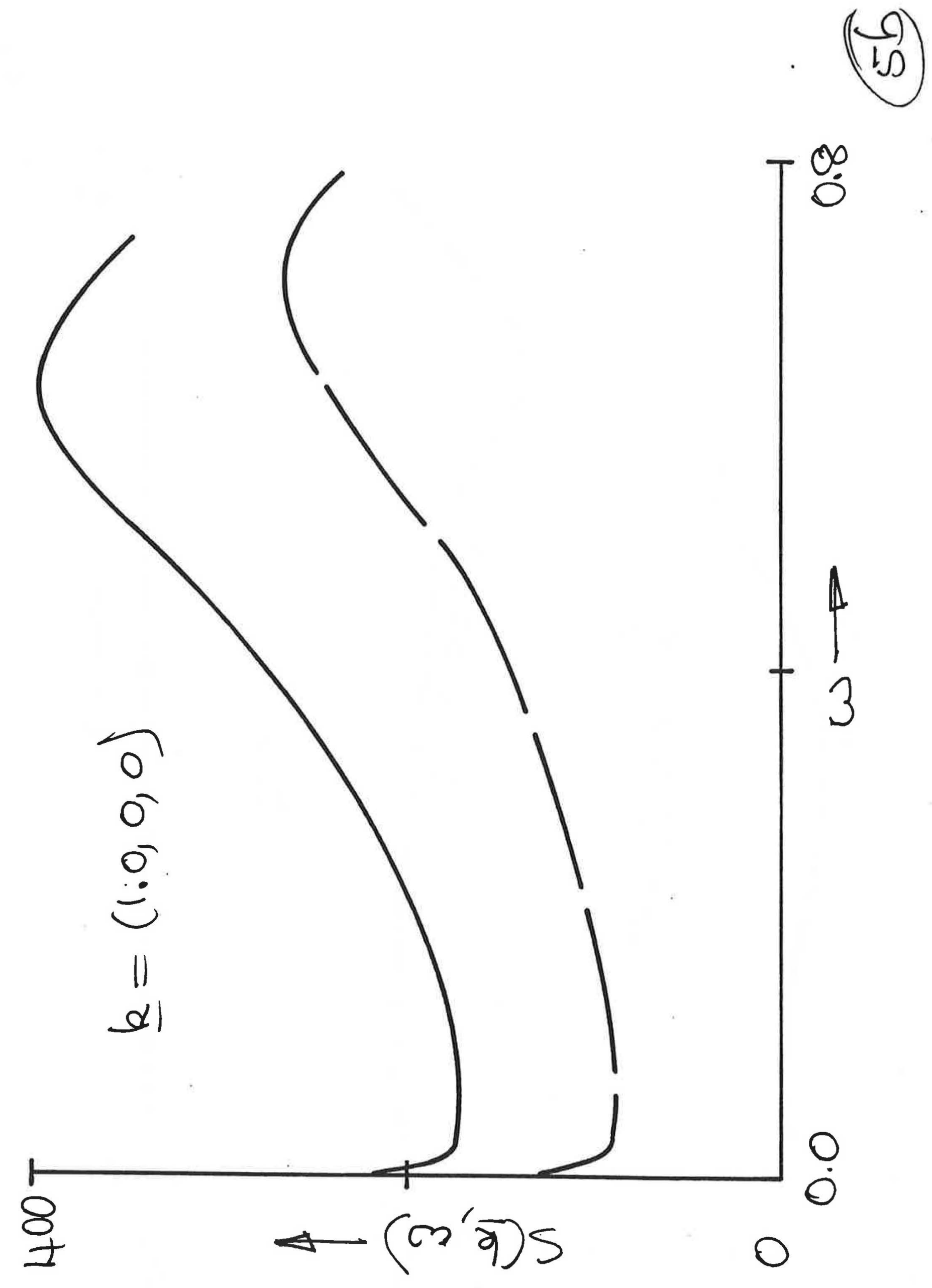




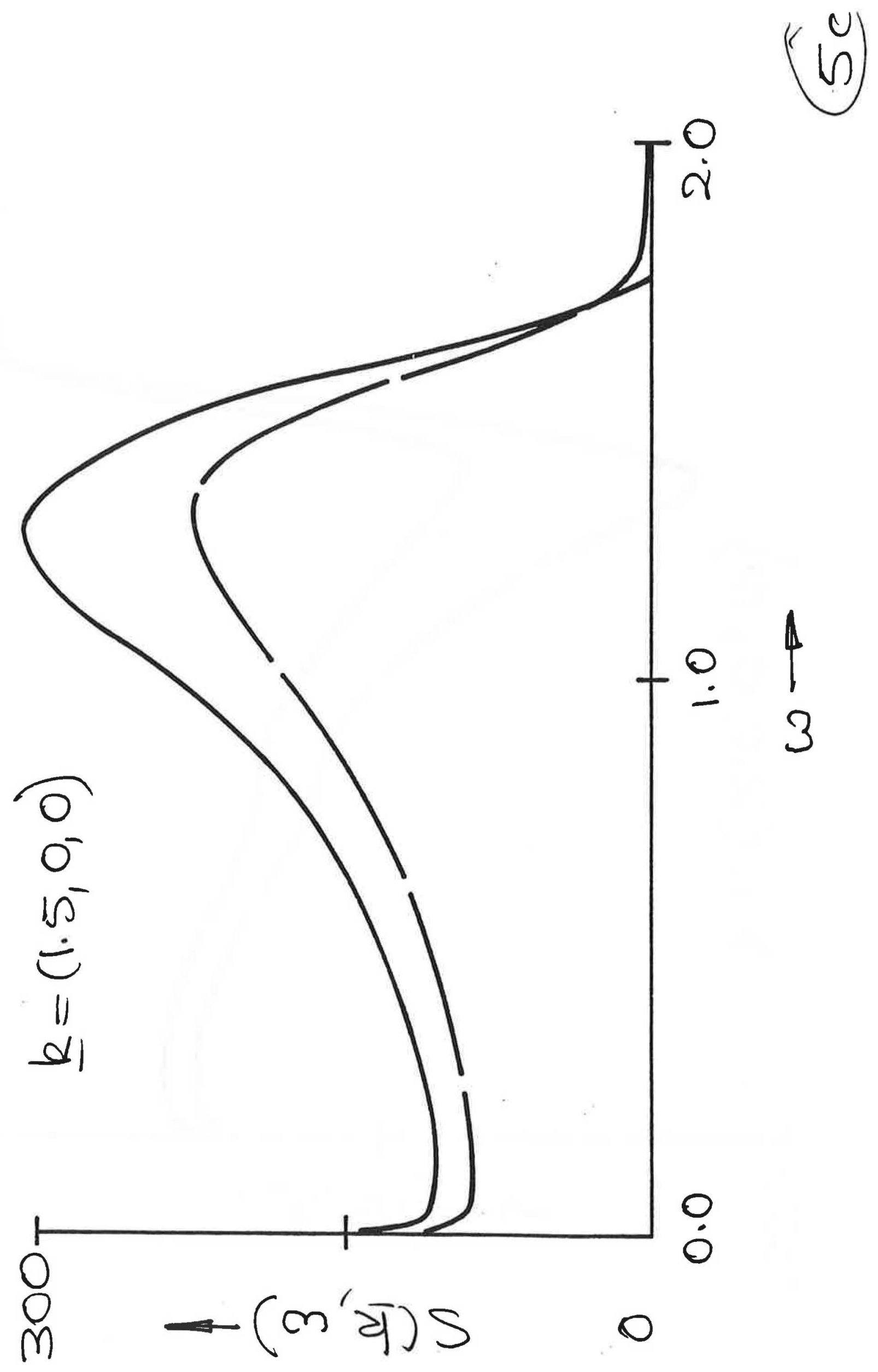


(18)

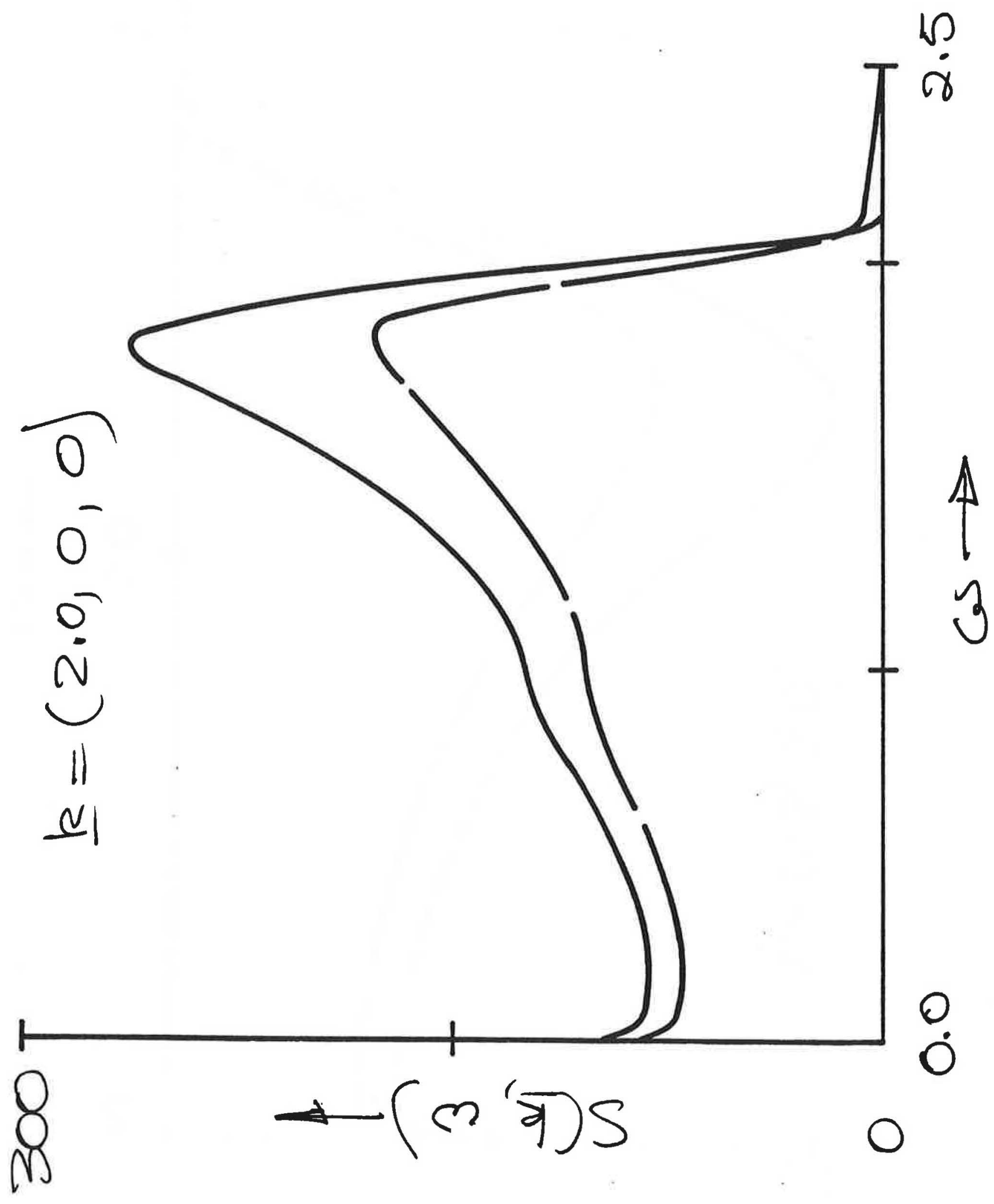


(3)

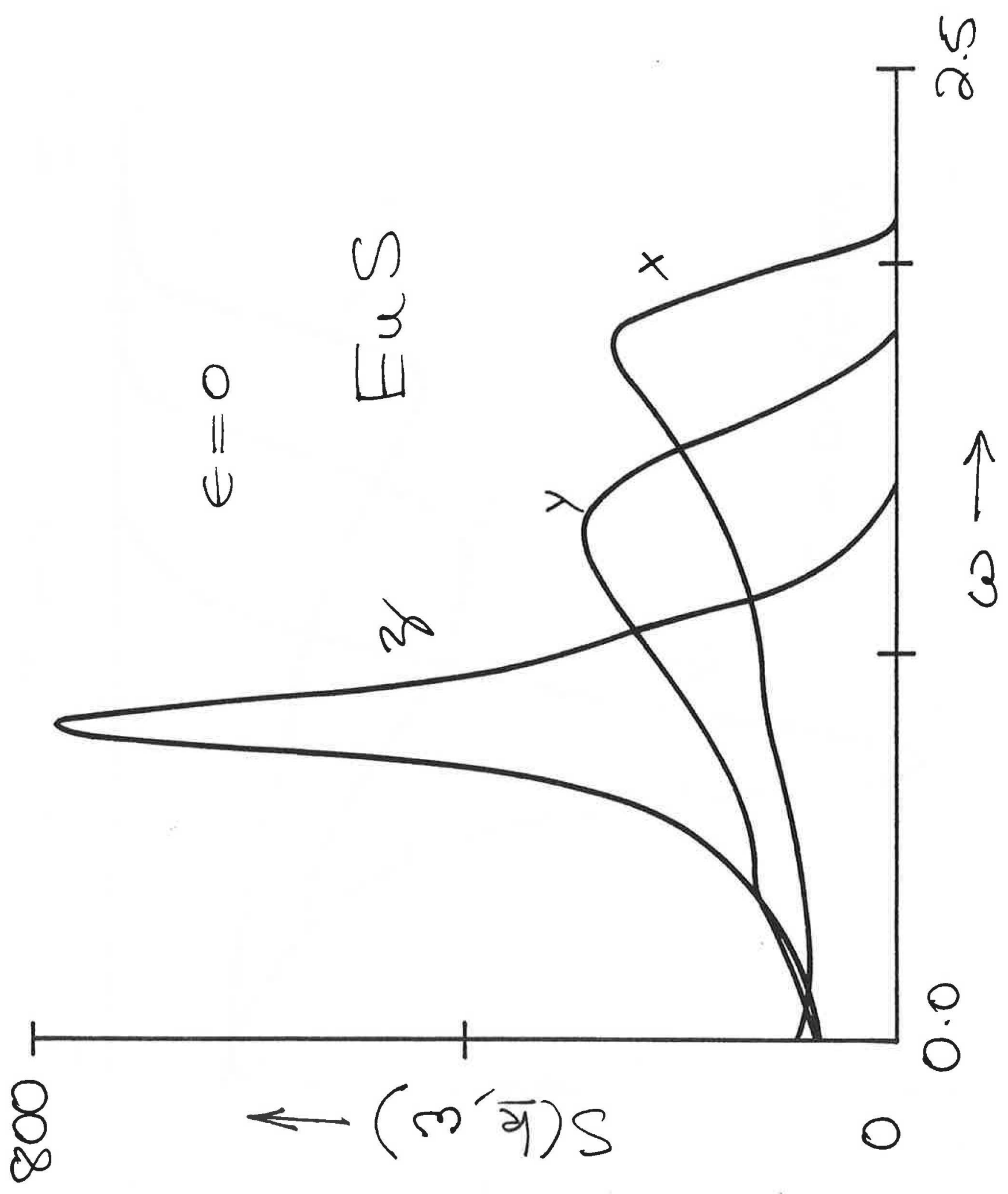


(v)

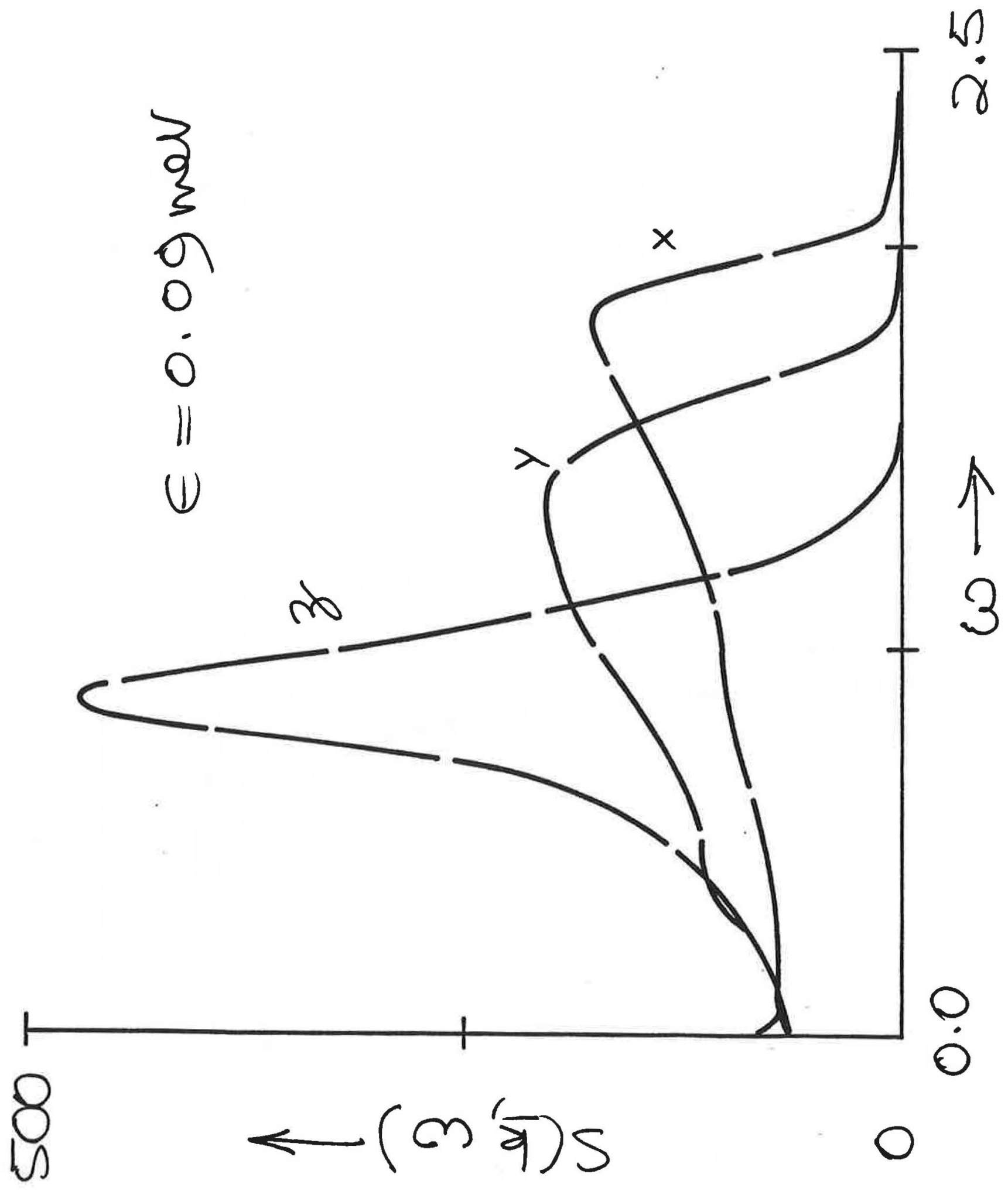





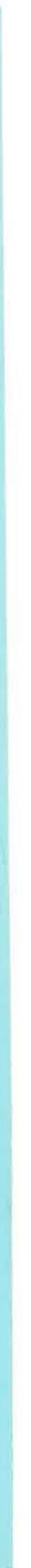

